\title{
Resultados obtidos em mulheres portadoras de endometriose pélvica avançada e mulheres com ligadura tubária, submetidas a ICSI: análise comparativa
}

\author{
Outcome of women with advanced pelvic endometriosis and women with tubal sterilization \\ submitted to ICSI: a comparative analysis \\ Tsutomu Aoki ${ }^{1}$, Nilson Donadio ${ }^{2}$, Antonio Pedro Auge ${ }^{3}$, Roberto Adelino de Almeida Prado ${ }^{4}$, \\ Edson Borges Junior ${ }^{5}$, Paulo Augusto Ayroza G. Ribeiro ${ }^{6}$, Roberto Euzébio dos Santos ${ }^{7}$
}

\section{RESUMO}

Objetivo: análise comparativa dos resultados obtidos em mulheres portadoras de endometriose pélvica avançada e mulheres com ligadura tubária, submetidas a injeção intracitoplasmática de espermatozóide (ICSI). Métodos: noventa e três mulheres com diagnóstico de infertilidade, com ciclo menstrual normal, não submetidas a qualquer tratamento hormonal e cirúrgico durante o último ano, índice de massa corporal igual a 20-25, portadoras de ovários sem a presença de cistos ou tumores participaram deste estudo e foram divididas em dois grupos: tubário (TUB), 39 mulheres com ligadura tubária, e endometriose (EDT), 54 mulheres com EDT-III e EDT-IV, submetidas a indução ovulatória com FSH-r e ICSI. Os dados clínicos e laboratoriais foram comparados. Testes $\chi^{2}$, Fisher, $t$ pareado, $t$ de Student e Mann-Whitney foram empregados. Resultados: níveis mais baixos de estradiol (2243,1 vs $1666,3 ; \mathrm{p}=0,001)$ e número menor de folículos por paciente $(16,9 v s 13,9 ; \mathrm{p}=0,001)$ foram recuperados no grupo EDT, apesar da utilização de maior número de unidades de FSH-r $(1775,6$ vs 1998,6; $\mathrm{p}=0,007$, para TUB e EDT, respectivamente). Não houve diferença quanto à taxa de recuperação ovocitária $(69 v s 73,5 \% ; \mathrm{p}=0,071)$ assim como nas taxas de fertilização normal $(83,7 v s$ $81,7 \% ; \mathrm{p}=0,563$ respectivamente para TUB e EDT. No entanto, menor número de pré-embriões ótimos foi obtido no grupo EDT $(36,4 v s 24,8 \%$, respectivamente para TUB e EDT; $\mathrm{p}=0,005)$. As taxas de gestação total $(41,0 v s 42,6 \% ; \mathrm{p}=0,950)$ e de implantação $(13,9 \%$ x 14,5\%; $\mathrm{p}=0,905)$ não foram significativamente diferentes quando TUB e EDT foram comparados. Conclusões: os ovários das mulheres do grupo EDT parecem ser menos responsivos à indução da ovulação com FSH-r. A endometriose parece comprometer o número médio de folículos e de pré-embriões ótimos sem prejudicar as taxas de recuperação ovocitária e fertilização. No entanto, uma vez formados, os pré-embriões do grupo EDT exibem chances de implantação e gestação semelhantes àquelas do grupo TUB.

PALAVRAS-CHAVE: Endometriose/terapia; Infertilidade feminina; Fertilização in vitro; Injeção de esperma intracitoplasmáticas; Medicina reprodutiva

\section{ABSTRACT}

Purpose: comparative analysis of the outcome of women with advanced pelvic endometriosis and women with tubal sterilization submitted to intracytoplasmic sperm injection (ICSI). Methods: ninety-three infertile women, with normal menstrual cycle, without hormonal or surgical treatment during 12 months, body mass index of 20-25, ovaries with no tumors or cysts were included in the present study and divided into two groups: tubal sterilization (TUB), 39 women, and endometriosis (EDT), 54 women with III-EDT and IV-EDT, undergoing ovulatory induction using r-FSH and ICSI. Clinical and laboratorial data were compared. $\chi^{2}$, Fisher, Student's $t$, and Mann-Whitney tests were employed. Results: lower estradiol levels $(2,243.1$ vs $1,666.3 ; \mathrm{p}=0.001)$ and lower number of follicles per patient ( $16.9 v s 13.9, \mathrm{p}=0.001)$ were noted in EDT group, in spite of more units of r-FSH (1,775.6 vs 1,998.6; $\mathrm{p}=0.007$, for TUB and EDT, respectively). There were no differences in the rates of retrieved oocyte $(69 v s 73.5 \%$; $\mathrm{p}=0.071)$ as well as in normal fertilization rates $(83.7 v s 81.7 \%$; $\mathrm{p}=0.563$, for TUB and EDT, respectively. However, lower number of top quality preembryos were obtained in patients from EDT group ( 36.5 vs $24.8 \%$, TUB and EDT, respectively; $\mathrm{p}=0.005)$. Total pregnancy ( $41.0 v s 42.6 \%$; $\mathrm{p}=0.950$ ) and implantation rates (13.9 vs 14.5\%; $\mathrm{p}=0.905)$ were not different when groups TUB and EDT were compared. Conclusions: ovaries of

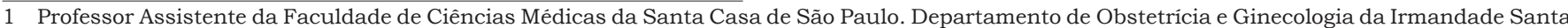
Casa de Misericórdia de São Paulo - São Paulo (SP) - Brasil; Diretor do Departamento de Obstetrícia e Ginecologia da Faculdade de Ciências Médicas da Irmandade Santa Casa de São Paulo. Departamento de Obstetrícia e Ginecologia da Santa Casa de Misericórdia de São Paulo - São Paulo (SP) - Brasil.

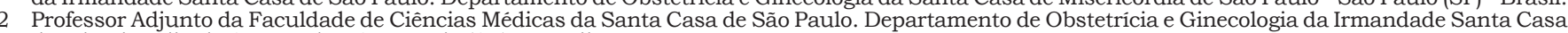
de Misericórdia de São Paulo - São Paulo (SP) - Brasil.

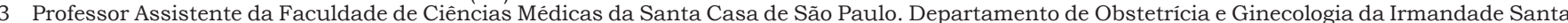
Casa de Misericórdia de São Paulo - São Paulo (SP) - Brasil.

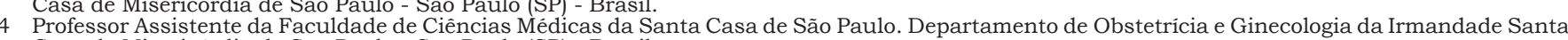
Casa de Misericórdia de São Paulo - São Paulo (SP) - Brasil.

5 Diretor do Fertility - Centro de Fertilização Assistida, São Paulo (SP) - Brasil.

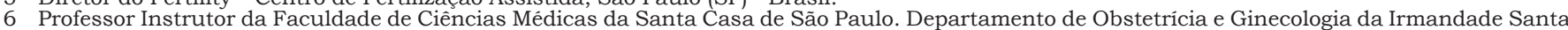
Casa de Misericórdia de São Paulo - São Paulo (SP) - Brasil.

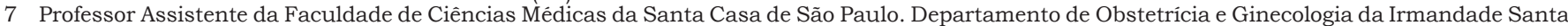
Casa de Misericórdia de São Paulo - São Paulo (SP) - Brasil;

Correspondência: Tsutomu Aoki

Rua Epeira, 185 - V. Madalena - 05447-020 - São Paulo - SP - Fone: (11) 3813-6783; Fax: (11) 3256-9633 - e-mail: draoki@uol.com.br

Recebido em: 4/3/2005 Aceito com modificações em: 17/11/2005 
women from EDT group seem to be less responsive to ovulatory induction with r-FSH. EDT seems to impair the mean number of follicles and top quality preembryos with no impairment of retrieved oocyte and fertilization rates. However, once obtained, preembryos from EDT patients are able to exhibit similar implantation potential and pregnancy when compared with patients from TUB group.

KEYWORDS: Endometriosis/therapy; Infertility, female; Fertilization in vitro; Sperm injections, intracytoplasmic; Reproductive medicine

\section{Introdução}

A endometriose (EDT), tecido endometrial ectópico constituído de glândulas e/ou estroma causado por fluxo sangüíneo retrógrado pelas tubas uterinas, foi inicialmente definida por Sampson ${ }^{1}$. Apesar da vasta literatura científica, aspectos relacionados ao tratamento da EDT são contraditórios ou inadequados. Apesar de ser doença comum que acomete cerca de 2 a $22 \%$ das mulheres em idade reprodutiva, é talvez, do ponto de vista ginecológico, a mais enigmática. Pode estar localizada em diferentes regiões (peritoneal, ovariana ou retovaginal), caracterizando doenças com etiopatogenias, sinais e sintomas distintos e, conseqüentemente, com tratamentos específicos ${ }^{2}$.

Uma das dúvidas que persiste até a presente data na literatura médica é encontrar a forma mais eficaz para se fazer o tratamento de mulheres portadoras de EDT associada à infertilidade, presente em 30 a $40 \%$ dos casos.

No entanto, desde o nascimento do primeiro bebê após a fertilização in vitro - FIV ${ }^{3}$ e posteriormente, após o relato da primeira gestação e nascimento de um bebê após a injeção intracitoplasmática de espermatozóide (ICSI) ${ }^{4}$, as técnicas de reprodução humana assistida (TRA) têm sido consideradas boas alternativas para manejar a infertilidade em pacientes portadoras de EDT avançada, possibilitando a ocorrência da gestação com nascimento por transferência embrionária em 22,7 a $42,3 \%$ dos $\operatorname{casos}^{5}$. Os resultados de gravidez em pacientes com EDT não são diferentes dos resultados das pacientes com infertilidade de causa tubária quando submetidas às TRA ${ }^{6}$.

Em outro estudo ${ }^{7}$ foram comparados os resultados obtidos em mulheres com EDT nos estádios III e IV e em mulheres com fator tubário submetidas à FIV, demonstrando-se condições desfavoráveis nas portadoras da EDT.

Mulheres portadoras de EDT associada à infertilidade submetidas a FIV respondem com niveis menores de todos os marcadores reprodutivos (19\% nos estádios I e II e 36,5\% nos estádios avançados). Niveis mais baixos de estradiol, assim como taxas significativamente mais baixas de gestação (13,8\%), foram encontradas e comparadas com um grupo de mulheres com ligadura tubária $(27,7 \%)$. Os autores reforçam a idéia de que a EDT não compromete exclusivamente a receptividade do endométrio mas também o desenvolvimento dos ovócitos e pré-embriões ${ }^{8}$. Um estudo por meta-análise mostrou resultado de $64,3 \%$ de gestação após a ICSI e 37,4\% após FIV 9 . No entanto, o estudo ressalta que os resultados de FIV dependeram também da quantidade e qualidade dos espermatozóides utilizados.

No entanto, não se sabe se submeter pacientes portadoras de EDT ou outras enfermidades ginecológicas a cirurgia prévia às TRA poderia melhorar os resultados dos procedimentos. Assim, Marconi et al. ${ }^{10}$ demonstram que a exérese de endometriomas não afeta os resultados da FIV quando comparados com o fator tubário isolado. Haveria diferença somente quanto à quantidade de ampolas de gonadotrofinas utilizadas durante o estímulo ovariano. Outros ${ }^{11}$, no entanto, observaram que cirurgias para endometrioma não melhoram as respostas da indução ovulatória ou da implantação embrionária, e, sendo assim, não devem ser utilizadas como rotina em mulheres assintomáticas, pois a ressecção de endometriomas poderia reduzir o tecido ovariano normal ${ }^{12,13}$.

Tendo como base a literatura que envolve a EDT e o dificil manejo da infertilidade associada à endometriose pélvica avançada, este trabalho teve por objetivo analisar o comprometimento ovariano e endometrial, assim como a qualidade embrionária e as chances de sucesso para conseguir uma gestação após o emprego da técnica de ICSI para este grupo de pacientes.

\section{Pacientes e Métodos}

Este estudo prospectivo foi realizado no período de janeiro de 1997 a dezembro de 2003. Os procedimentos foram realizados na Clínica Fertility - Centro de Fertilização Assistida - São Paulo, em intercâmbio com a Faculdade de Ciências Médicas da Santa Casa de São Paulo, Serviço de Reprodução Humana e Laboratório de Reprodução Assistida do Departamento de Obstetrícia e Ginecologia - DOGi da Santa Casa de Misericórdia de São Paulo e Pró-Embryo - Técnicas Avançadas em Reprodução Humana. O projeto de pesquisa foi aprovado pela Comissão de Ética em Pesquisa da Santa Casa. Todos os casais assinaram o termo de 
consentimento pós-informado de acordo com a Resolução $\mathrm{n}^{\circ} 1358$ do Conselho Federal de Medicina (CFM, 1992), autorizando a utilização dos dados clínicos e laboratoriais.

De um total de 2.774 casais avaliados neste periodo, foram selecionadas para este estudo 54 mulheres inférteis, portadoras de endometriose pélvica avançada - estádios III e IV e, paralelamente, 39 mulheres com ligadura tubária, cirurgia esta realizada em outros serviços.

Os critérios de inclusão considerados para este estudo foram: mulheres hígidas com diagnóstico de infertilidade, ciclos menstruais normais, portadoras de EDT avançada (estádios III ou IV) segundo a Classificação da Sociedade Americana de Medicina Reprodutiva ${ }^{14}$; com índice de massa corporal em torno de 20 a 25 e sem a presença de cistos ou tumores ovarianos.

Os critérios de exclusão considerados para este estudo foram: baixa reserva ovariana caracterizada pela dosagem de estradiol sérico (E2) e do FSH no terceiro dia do ciclo menstrual anterior ao do procedimento; diagnóstico de fator uterino cervical e/ou fator uterino corporal após exame radiológico, histerossalpingografia e ultra-sonografia pélvica transvaginal e/ou parceiros com alterações na análise seminal e/ou com distúrbios ejaculatórios ou de coleta.

Após propedêutica para investigação anatômica e funcional, as 93 mulheres selecionadas foram divididas em dois grupos: TUB total, constituído por 39 mulheres que apresentavam ligadura tubária, sem nenhum outro fator causador de infertilidade, e EDT total, 54 mulheres portadoras de endometriose pélvica avançada. Ambos os grupos foram posteriormente divididos em dois subgrupos de acordo com a faixa etária, sendo TUB idade menor (19 pacientes) e EDT idade menor (38 pacientes) compostos por mulheres com idade igual ou inferior a 35 anos e TUB idade maior (20 pacientes) e EDT idade maior (16 pacientes) com idade superior a 35 anos.

Em todos os casos empregou-se protocolo longo para estimulação folicular com bloqueio hipofisário utilizando os análogos do fator liberador de gonadotrofina (GnRH-a) desde o $21^{\circ}$ dia do ciclo anterior ao do procedimento, com aplicação subcutânea de $1,0 \mathrm{mg}(0,2 \mathrm{~m})$ por dia de acetato de leuprolida (Lupron - $\mathrm{Abbott}^{\circledR}$ ) até o dia da menstruação.

No segundo dia do ciclo menstrual a ser estudado foi dosado o E2 por radioimunoensaio, a fim de confirmar o bloqueio hipofisário. O valor deveria ser inferior ou igual a $30 \mathrm{pg} / \mathrm{mL}$; nesta ocasião se reduziu a dose do acetato de leuprolida 0,5 $\mathrm{mg}(0,1 \mathrm{~mL}$ por dia até a aplicação por via intramuscular da gonadotrofina coriônica humana (hCG) em dose única de 10.000 UI (Profasi HP 10.000 UI - Serono ${ }^{\circledR}$.
Sob bloqueio hipofisário, a estimulação ovariana foi iniciada pela aplicação diária via subcutânea ou intramuscular, com doses fixas de FSH recombinante (FSH-r) de $225 \mathrm{UI} /$ dia (Gonal-F Serono $\left.^{\circledR}\right)$, a partir do $3^{\circ}$ dia do ciclo menstrual. No $8^{\circ}$ dia do ciclo menstrual iniciou-se o controle ultra-sonográfico endovaginal seriado com a adaptação das doses subseqüentes de FSH-r em função da resposta ovariana.

A monitorização ovariana ultra-sonográfica foi realizada a partir do $8^{\circ}$ dia do ciclo menstrual, em estudo em dias alternados até se atingir diâmetro folicular médio de 18 a $20 \mathrm{~mm}$, quando foi orientada a aplicação de gonadotrofina coriônica. A espessura endometrial foi medida pela ultrasonografia pélvica endovaginal seriada, devendo ser trilaminar no período estrogênico. O E2 foi dosado por radioimunoensaio em dias alternados a partir do $8^{\circ}$ dia, a fim de controlar a superovulação e evitar a ocorrência da sindrome de hiperestimulação ovariana acentuada, sendo anotada a dosagem do E2 no dia da aplicação do hCG.

A aspiração folicular para a coleta de ovócitos realizou-se pela via transvaginal guiada pela ultrasonografia (Aloka ${ }^{\circledR} 500$ ), 34 a 36 horas após a administração do hCG. O procedimento foi executado sob sedação com propofol ${ }^{\circledR}$, por via endovenosa e com monitorização cardiorrespiratória. No fluido folicular, os ovócitos foram identificados e classificados ${ }^{15}$, sob aumento de $80 \mathrm{X}$ em lupa (SMZU Nikkon, Japão). Os ovócitos permaneceram incubados após a sua captura em meio de cultura HTF a $37^{\circ} \mathrm{C}, 5,0 \%$ de $\mathrm{CO}_{2}$, por um período entre 4 e 6 horas.

Decorrido este período, o complexo cumuluscorona foi removido pela colocação em solução de hialuronidase tipo VIII na concentração de $80 \mathrm{UI} /$ $\mathrm{mL}$, permitindo a classificação definitiva quanto ao grau de maturação (prófase, metáfase I ou metáfase II, MII). Ovócitos em MII foram considerados "gametas fertilizáveis" e depois da denudação (retirada das células do complexo cumulus-corona) foram transferidos do meio de incubação para gotas de $5 \mu \mathrm{L}$ do meio de cultura HTF/Hepes suplementados com $10 \%$ do soro da mulher ou de soro sintético substituto. Sob óleo mineral em microgotas foram micromanipulados para a ICSI, entre 4 e 6 horas após a aspiração folicular.

No dia da punção folicular, os parceiros foram orientados para a coleta por masturbação da amostra seminal em recipiente estéril, após abstinência de dois a cinco dias. Para a seleção dos espermatozóides do fluido seminal foram usados gradientes descontínuos de densidade (Sperm-Pre$100^{\mathrm{TM}}$ - Scandinavian IVF Science $\mathrm{AB}^{\circledR}$, Sweden), nas concentrações 40 e $90 \%$.

Realizou-se a micromanipulação de gametas - ICSI em microscópio invertido Nikkon ${ }^{\circledR}$ equipa- 
do com sistema de lentes Hoffman, e acoplado a micromanipuladores Narishige ${ }^{\circledR}$. Uma solução de $10 \%$ com polivinilpirrolidona (PVP-ICSI-100 (Scandinavian IVF Science $\mathrm{AB}^{\circledR}$, Sweden), diluída em IVF-50, foi usada para imobilizar os espermatozóides ${ }^{16}$. Utilizando-se a micropipeta apreensora (holding), o ovócito foi posicionado com o primeiro corpúsculo polar direcionado para 6:00 horas. Após sua imobilização em PVP e a desestabilização de seu flagelo, o espermatozóide foi aspirado pela micropipeta injetora. Em seguida, foi injetado no citoplasma do ovócito às $3: 00 \mathrm{~h}$, isto é, no eixo equatorial do ovócito, junto com pequeno volume de citoplasma ovocitário aspirado.

Os ovócitos micromanipulados foram mantidos em cultura e a fertilização foi confirmada cerca de 16 a 18 horas depois da ICSI (dia +1), pela presença ou ausência de pró-núcleos. Foi definida fertilização normal pela presença de dois prónúcleos (2PN) e a extrusão do segundo corpúsculo polar na fase de zigoto ou pró-nucleado. A presença de 1PN, 3PN ou mais foi considerada como fertilização anormal e a ausência de PNs foi classificada como falha de fertilização.

A ativação do ovócito foi observada pelo início de sucessivas divisões celulares - a clivagem. A transferência embrionária para a cavidade uterina ocorreu, sempre, no dia $+3(\mathrm{D}+3)$ após o procedimento da ICSI.

Para a classificação e seleção embrionária os pré-embriões foram continuamente observados desde a tarde do dia $+1(\mathrm{D}+1)$ até o momento da transferência ( $\mathrm{D}+3)$, com o intuito de acompanhar o desenvolvimento e a qualidade embrionária. No presente trabalho foi considerada somente a análise do embrião do dia D+3.

Os principais parâmetros levados em conta para seleção de pré-embriões no $\mathrm{D}+3$ foram aspectos morfológicos baseados em critérios previamente descritos ${ }^{17}$, em relação ao número de células, simetria dos blastômeros e porcentagem de fragmentação, a saber: IA - blastômeros simétricos sem fragmentação; IB - blastômeros simétricos com até $10 \%$ de fragmentação; IC - blastômeros simétricos com 11 a 20\% de fragmentação; ID - blastômeros simétricos com 20 a 35\% de fragmentação, IE - blastômeros simétricos com mais de $35 \%$ de fragmentação; IIA - blastômeros assimétricos sem fragmentação; IIB - blastômeros assimétricos com até $10 \%$ de fragmentação; IIC - blastômeros assimétricos com 11 a $20 \%$ de fragmentação; IID - blastômeros assimétricos com 20 a 35\% de fragmentação, IIE - blastômeros assimétricos com mais de 35\% de fragmentação.

De acordo com o protocolo empregado neste estudo, os pré-embriões que apresentaram clivagem normal (quatro a oito células) com classificação IA, IB ou IC foram considerados "ótimos".
Pré-embriões com clivagem normal (quatro a oito células) e subseqüente classificação como ID, IIA, IIB e IIC foram considerados "bons".

Foram transferidos rotineiramente até quatro embriões, conforme a Resolução $\mathrm{n}^{\circ} 1358$ do Conselho Federal de Medicina, após 72 horas em cultura. Previamente aplicava-se o protocolo de classificação e seleção embrionária, optando-se principalmente pelos pré-embriões "ótimos" e "bons".

O cateter utilizado para a transferência embrionária foi o de Frydman ou Wallace, sob orientação ultra-sonográfica, com repleção vesical com ingestão de quatro copos de água, 60 minutos previamente à transferência. Os pré-embriões foram injetados de forma lenta e gradual, assim que o cateter ultrapassasse o orificio interno do canal cervical.

As mulheres receberam, depois da transferência embrionária, progesterona sob a forma de óvulos vaginais de $200 \mathrm{mg}$ aplicados três vezes ao dia até o dia do teste de gravidez; tal conduta foi mantida até a $12^{\mathrm{a}}$ semana de gestação.

Foi considerada, como índice ou taxa de fertilização, a relação entre o número de ovócitos fertilizados e o número de ovócitos intactos; como índice ou taxa de implantação, a relação do número de sacos gestacionais pelo número de préembriões transferidos; como de gestação, a relação de gestação clínica pelo número total de mulheres.

Foram utilizados os testes $\chi^{2}$ ou teste exato de Fisher para comparar variáveis categóricas. Para variáveis continuas e dependentes foi empregado o teste $t$ pareado e, para variáveis contínuas e independentes, foram utilizados o teste $t$ de Student e/ou Mann-Whitney. O resumo estatístico incluiu média e desvio padrão com intervalo de confiança de $95 \%$.

\section{Resultados}

A média da idade ( \pm desvio-padrão) das mu-

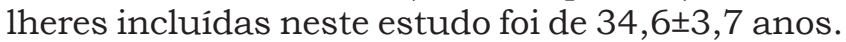
Não houve diferença quando os grupos totais foram comparados: $35,2 \pm 3,4$ vs $34,1 \pm 3,8$ anos, respectivamente para TUB total e EDT total; $p=0,498$. O mesmo foi notado nos grupos divididos pela idade da paciente $(32,3 \pm 1,9$ vs $32,3 \pm 2,4$ anos, respectivamente para TUB idade menor e EDT idade menor; $\mathrm{p}=0,965$, e $37,9 \pm 1,9$ us $38,6 \pm 2,7$ respectivamente para TUB idade maior e EDT idade maior; $\mathrm{p}=0,427)$.

Em relação ao FSH-r, foi possivel notar que foram necessárias mais unidades durante a indução ovulatória nas pacientes com EDT tanto 
no grupo total $(1775,6 \pm 377,1$ vs $1998,6 \pm 391,9$, respectivamente para grupo EDT total $v$ s TUB total; $\mathrm{p}=0,007)$ quanto nos grupos separados em relação à idade $(1676,3 \pm 326,5$ vs $1895,4 \pm 349,5$, respectivamente para TUB idade menor $v s$ EDT idade menor; $p=0,025$, e $1870,0 \pm 405,1$ vs $2243,7 \pm 387,8$, respectivamente para TUB idade maior $v$ S EDT idade maior; $\mathrm{p}=0,008$ ).

Em contrapartida, os níveis de E2 apresentaram-se significativamente mais baixos na presença de EDT $(2243,1 \pm 638,2$ vs $1666,3 \pm 475$, 3 respectivamente para TUB total $v$ sDT total; $p=0,001$ ) tanto nas pacientes mais jovens $(2674,0 \pm 599,0$ vs $1820,3 \pm 456,9$, respectivamente para TUB idade menor vs EDT idade menor; $\mathrm{p}=0,001)$ quanto naquelas com idade maior que 35 anos $(1833,6 \pm 334,9$ vs $1300,4 \pm 279,3$, respectivamente para TUB idade maior $v$ s EDT idade maior; $\mathrm{p}=0,001$ ).

Houve diferença em relação ao número médio de folículos puncionados por mulher tanto no grupo total $(16,9 \pm 3,3$ vs $13,9 \pm 3,7$, respectivamente para TUB $v s$ EDT; $\mathrm{p}=0,001$ ) quanto no grupo de pacientes com idade superior a 35 anos $(16,8 \pm 2,8$ vs $10,6 \pm 2,2$, respectivamente para TUB vs EDT; $\mathrm{p}=0,001)$. No entanto, o mesmo não ocorreu no grupo de pacientes mais jovens $(17,1 \pm 3,8$ vs $15,4 \pm 3,2$, respectivamente para TUB idade menor $v$ s EDT idade menor; $\mathrm{p}=0,101$ ).

Quanto à taxa de recuperação ovocitária (isto é, a relação entre o número de ovócitos obtidos por folículos puncionados), não foi encontrada diferença em nenhum dos grupos analisados (69 vs $73,5 \%$, respectivamente para TUB total; e EDT total; $\mathrm{p}=0,071,70,1$ vs $74,0 \%$ para TUB idade menor vs EDT idade menor, respectivamente; $p=0,240$, e 67,9 versus $71,6 \%$, respectivamente para TUB idade maior vs EDT idade maior; $p=0,449$.

Um maior número de ovócitos maduros, em MII, foram obtidos no grupo total $(83,7$ vs $77,6 \%$, respectivamente para TUB total $v$ s EDT total; $\mathrm{p}=0.017)$ e no subgrupo de pacientes com idade superior a 35 anos $(90,3$ us $71,6 \%$, respectivamente para TUB idade maior vs EDT idade maior; $p=0,001)$. Não houve diferença quanto aos resultados obtidos no subgrupo de pacientes mais jovens $(77,2$ vs $80,8 \%$, respectivamente para TUB idade menor $v s$ EDT idade menor; $p=0,316)$.

Maior porcentagem de ovócitos em MII foram recuperados no grupo TUB total $(83,7$ vs $77,6 \%$; respectivamente para TUB vs EDT; $p=0,017)$. Em contrapartida, a taxa de ovócitos fertilizados foi significativamente maior no grupo de pacientes com EDT, independentemente da idade $(78,0$ vs $82,6 \%$, respectivamente para TUB vs EDT; $\mathrm{p}=0,019)$. Não houve diferença estatística entre a porcentagem de ovócitos com fertilização normal e o número médio de embriões transferidos por paciente (Tabela 1).
Tabela 1 - Resultados laboratoriais e clínicos obtidos e comparados entre as pacientes com ligadura tubária (TUB) e portadoras de endometriose (EDT) independentemente da idade da mulher. Após a análise estatística, $p<0,05$ foi considerado significante.

\begin{tabular}{lccc}
\hline & TUB & EDT & p \\
\hline Folículos/ovócitos & $661 / 456$ & $754 / 554$ & \\
Ovócitos em MII (\%) & $382(83,7)$ & $430(77,6)$ & 0,017 \\
Ovócitos micromanipulados & 382 & 430 & \\
Ovócitos fertilizados (\%) & $298(78,0)$ & $355(82,6)$ & 0,019 \\
2PN (\%) & $242(83,7)$ & $290(81,7)$ & 0,563 \\
Média ET/mulher ( \pm desvio) & $3,5 \pm 0,7$ & $3,3 \pm 0,9$ & 0,124 \\
Pré-embriões ótimos/2PN (\%) & $88 / 242(36,4)$ & $72 / 290(24,8)$ & 0,005 \\
Pré-embriões bons/2PN (\%) & $35 / 242(14,5)$ & $32 / 290(11,0)$ & 0,291 \\
Pré-embriões ótimos/ET (\%) & $88 / 137(64,2)$ & $72 / 172(41,9)$ & 0,078 \\
Pré-embriões bons/ET (\%) & $35 / 137(25,5)$ & $32 / 172(18,6)$ & 0,183 \\
Gestação total / mulher (\%) & $16 / 39(41,0)$ & $23 / 54(42,6)$ & 0,950 \\
Gestação em evolução (\%) & $10 / 16(62,5)$ & $13 / 23(56,5)$ & 0,661 \\
Taxa de implantação (\%) & 13,9 & 14,5 & 0,905
\end{tabular}

Folículos/ovócitos: número de ovócitos recuperados pelo total de folículos puncionados; MIl: ovócitos em metáfase II; 2PN: taxa de fertilização normal; ET: embriões transferidos.

Após a classificação morfológica dos préembriões, maior quantidade de pré-embriões foram classificados como "ótimos" em relação ao número total de ovócitos que apresentaram fertilização normal no grupo TUB $(36,4$ vs $24,8 \%$, respectivamente para grupos TUB e EDT; $p=0,005$ ). No entanto, não houve diferença quando esta taxa foi calculada em relação ao número total de embriões selecionados para a transferência. A quantidade de pré-embriões classificados como "bons" foi semelhante em ambos os grupos em relação ao número de ovócitos fertilizados e ao número de embriões transferidos. As taxas de gestação e de implantação foram semelhantes quando os grupos foram comparados (Tabela 1).

Quando as pacientes com idade inferior ou igual a 35 anos foram analisadas, os resultados obtidos em relação ao número de ovócitos em MII, taxa de fertilização total, taxa de fertilização normal e número médio de embriões transferidos por paciente não foram diferentes entre os grupos (Tabela 2).

Entretanto, maior porcentagem de pré-embriões "ótimos" foi obtida em relação ao número de $2 \mathrm{PN}$ (35,0 vs 20,7\%, respectivamente para TUB e EDT idade menor; $p=0,005)$ e também em relação ao número de embriões transferidos $(63,1$ vs $41,1 \%$, respectivamente para TUB e EDT idade menor; $p=0,005$ ). Quando a porcentagem de "bons" pré-embriões foi calculada em relação ao número de embriões transferidos, significância estatística também foi observada $(27,7$ vs $18,7 \%$, respectivamente para TUB e EDT idade menor; $p=0,020$ ). No entanto, semelhantes taxas de gestação e implantação foram obtidas em pacientes do grupo TUB e EDT (Tabela 2). 
Tabela 2 - Resultados laboratoriais e clínicos obtidos e comparados entre as pacientes com ligadura tubária (TUB) e portadoras de endometriose (EDT) com idade inferior ou igual a 35 anos. Após a análise estatística, $p<0,05$ foi considerado significante.

$$
\text { TUB EDT } p
$$

idade menor idade menor

\begin{tabular}{lccc}
\hline Folículos/ovócitos & $325 / 228$ & $585 / 433$ & \\
Ovócitos em MII (\%) & $176(77,2)$ & $350(80,8)$ & 0,316 \\
Ovócitos micromanipulados & 176 & 350 & \\
Ovócitos fertilizados (\%) & $144(81,8)$ & $302(86,3)$ & 0,223 \\
2PN & $117(81,2)$ & $242(80,1)$ & 0,880 \\
Média ET/mulher ( \pm desvio) & $3,4 \pm 0,7$ & $3,2 \pm 0,8$ & 0,875 \\
Pré-embriões ótimos/2PN (\%) & $41 / 117(35,0)$ & $50 / 242(20,7)$ & 0,005 \\
Pré-embriões bons/2PN (\%) & $18 / 117(15,4)$ & $23 / 242(9,5)$ & 0,142 \\
Pré-embriões ótimos/ET (\%) & $41 / 65(63,1)$ & $50 / 123(41,1)$ & 0,005 \\
Pré-embriões bons/ET (\%) & $18 / 65(27,7)$ & $23 / 123(18,7)$ & 0,020 \\
Gestação total/mulher (\%) & $8 / 19(42,1)$ & $17 / 38(44,7)$ & 0,924 \\
Gestação em evolução (\%) & $6 / 8(75,0)$ & $10 / 17(58,8)$ & 0,661 \\
Taxa de implantação (\%) & 13,1 & 15,4 & 0,909
\end{tabular}

Folículos/ovócitos: número de ovócitos recuperados pelo total de folículos puncionados; MIl: ovócitos em metáfase II; 2PN: taxa de fertilização normal; ET: embriões transferidos.

No grupo de pacientes com idade superior a 35 anos, a presença de EDT comprometeu as taxas de recuperação de ovócitos em MII (90,3 vs $71,6 \%$, respectivamente para TUB e EDT idade maior; $\mathrm{p}=0,001)$, de fertilização total $(74,8$ us $66,2 \%$, respectivamente para TUB e EDT idade maior; $\mathrm{p}=0,001)$ e a porcentagem de pré-embriões ótimos em relação ao número de embriões transferidos $(65,3$ vs $44,9 \%$, respectivamente para TUB e EDT idade maior; $p=0,041)$. Não houve diferença entre os grupos quando os demais parâmetros foram comparados (Tabela 3).

Tabela 3 - Resultados laboratoriais e clínicos obtidos e comparados entre as pacientes com ligadura tubária (TUB) e portadoras de endometriose (EDT) com idade superior a 35 anos. Após a análise estatística, $p<0,05$ foi considerado significante.

\begin{tabular}{lccc}
\hline & TUB & EDT & $p$ \\
& idade maior & idade maior & \\
\hline Folículos/ovócitos & $336 / 228$ & $169 / 121$ & \\
Ovócitos em MIl (\%) & $206(90,3)$ & $121(71,6)$ & 0,001 \\
Ovócitos micromanipulados & 206 & 121 & \\
Ovócitos fertilizados & $154(74,8)$ & $80(66,2)$ & 0,001 \\
2PN & $125(81,2)$ & $50(94,3)$ & 0,168 \\
Média ET/mulher ( \pm desvio) & $3,6 \pm 0,7$ & $3,1 \pm 1,4$ & 0,091 \\
Pré-embriões ótimos/2PN (\%) & $47 / 125(37,6)$ & $22 / 50(44,0)$ & 0,414 \\
Pré-embriões bons/2PN (\%) & $17 / 125(13,6)$ & $9 / 50(18,0)$ & 0,541 \\
Pré-embriões ótimos/ET (\%) & $47 / 72(65,3)$ & $22 / 49(44,9)$ & 0,041 \\
Pré-embriões bons/ET (\%) & $17 / 72(23,6)$ & $9 / 49(18,4)$ & 0,642 \\
Gestação total/mulher (\%) & $8 / 20(40,0)$ & $6 / 16(37,5)$ & 0,848 \\
Gestação em evolução (\%) & $4 / 8(50,0)$ & $3 / 6(50,0)$ & 1,000 \\
Taxa de Implantação (\%) & 12,5 & 12,2 & 0,884 \\
\hline
\end{tabular}

Folículos/ovócitos: número de ovócitos recuperados pelo total de folículos puncionados; MII: ovócitos em metáfase II; 2PN: taxa de fertilização normal; ET: embriões transferidos.
Interessantemente, melhores pré-embriões foram obtidos nas pacientes portadoras de EDT com idade superior a 35 anos quando a taxa calculada baseou-se no número de embriões que exibiram fertilização normal $(20,7$ vs 44,0\%, respectivamente, de ótimos embriões em EDT idade menor us EDT idade maior; $p=0,002 ; 9,5$ vs $18,0 \%$, respectivamente, de bons embriões em EDT idade menor vs EDT idade maior; $p=0,012)$. Significância estatística não foi notada quando os demais parâmetros analisados foram comparados entre pacientes mais jovens e mais velhas portadoras de EDT (Tabela 4).

Tabela 4 - Resultados laboratoriais e clínicos obtidos e comparados entre as pacientes portadoras de endometriose (EDT) de diferentes faixas etárias (EDT idade menor com pacientes $\leq 35$ anos e EDT idade maior com $>35$ anos). Após a análise estatística, $p<0,05$ foi considerado significante.

\begin{tabular}{lccc}
\hline & $\leq 35$ anos & $>35$ anos & p \\
\hline Recuperação ovocitária (\%) & 74,0 & 71,6 & 0,569 \\
Ovócitos em MII & 80,8 & 71,6 & 0,112 \\
Ovócitos fecundados (\%) & 86,3 & 66,2 & 0,087 \\
2PN (\%) & 80,1 & 94,3 & 0,356 \\
Média ET/mulher & $3,2 \pm 0,6$ & $3,1 \pm 1,4$ & 0,087 \\
Pré-embriões ótimos/2PN (\%) & 20,7 & 44,0 & 0,002 \\
Pré-embriões bons/2PN (\%) & 9,5 & 18,0 & 0,012 \\
Pré-embriões ótimos/ET (\%) & 41,1 & 44,9 & 0,735 \\
Pré-embriões bons/ET(\%) & 18,7 & 18,4 & 0,867 \\
Gestação total (\%) & 44,7 & 37,5 & 0,849 \\
Gestação em evolução (\%) & 58,8 & 50,0 & 1,000 \\
Taxa de implantação (\%) & 15,4 & 12,2 & 0,765 \\
\hline
\end{tabular}

Recuperação ovocitária: porcentagem de ovócitos recuperados pelo total de folículos puncionados; MII: ovócitos em metáfase II; 2PN: taxa de fertilização normal; ET: embriões transferidos.

\section{Discussão}

Os resultados obtidos após a FIV com a técnica de ICSI em mulheres inférteis portadoras de endometriose avançada foram obtidos e comparados com mulheres após ligadura tubária.

Demonstrou-se ${ }^{18}$ que, independentemente do estádio da EDT, gestações espontâneas ocorrem em $37 \%$ dos casos com estadiamento I e II, em $22 \%$ no estádio III e em apenas 3\% dos casos no estádio IV, de acordo com a classificação da Associação Americana de Medicina Reprodutiva ${ }^{14}$.

As pacientes do grupo EDT necessitaram mais UI de FSH-r durante o hiperestimulo ovariano controlado quando comparadas com as do grupo TUB. Mesmo assim, níveis mais baixos de estradiol foram obtidos. Estudo comparativo de FIV em pacientes portadoras de EDT demonstrou que todos os parâmetros reprodutivos em resposta aos estímulos de indução ovulatória estavam diminuídos em relação ao fator tubário, sugerindo que os ovários, nes- 
tas pacientes, parecem ser menos responsivos ${ }^{8}$.

Outro estudo ${ }^{19}$ demonstrou uma disfunção ovulatória acompanhada por uma insuficiência lútea em mulheres com EDT minima e leve após a utilização do hormônio liberador da tirotrofina, caracterizando hiperprolactinemia e niveis séricos menores de estradiol em até 36\% na fase folicular em relação a outras mulheres sem EDT.

Neste estudo, foi possivel notar que a presença de EDT parece interferir na quantidade de folículos recuperados após a punção ovariana, tanto no grupo total como no grupo de pacientes mais velhas. No entanto, não houve comprometimento em relação ao número de ovócitos recuperados por folículo (taxa de recuperação ovocitária).

Menos ovócitos em MII foram obtidos nas mulheres do grupo EDT total e EDT idade menor. Este fato assume importância na prática, pois orienta os profissionais na rotina para acelerarem as indicações da reprodução assistida em pacientes jovens com EDT avançada, tendo em vista a qualidade da resposta ovariana destes ovários, notada neste e em outros estudos ${ }^{19}$.

Foram notadas diferenças significativas no total de ovócitos fecundados, a favor do grupo EDT, muito embora o número de ovócitos recuperados em MII tenha sido menor. No entanto, deste total de ovócitos fertilizados, não houve diferença em relação às taxas de fertilização normal quando os grupos foram comparados. Entretanto, uma vez formado, o pré-embrião oriundo da paciente do grupo EDT exibe, aparentemente, o mesmo potencial de implantação visto que, apesar de menor número de pré-embriões "ótimos" ter sido transferido nestas pacientes, independentemente da idade, foram obtidas taxas de gestação semelhantes àquelas do grupo TUB.

As observações relacionadas aos ovócitos e pré-embriões relatadas anteriormente não se traduzem em relação às taxas de implantação e de gestação. No total, ocorreram 16 gestações entre as 39 mulheres do grupo TUB (41,0\%). Entre os casos de endometriose, houve 23 gestações em 54 mulheres (42,6\%). Houve evolução de 13 das 23 gestações do grupo EDT contra 10 de 16 do grupo TUB (56,5\%). Os indices de implantação não foram diferentes quando comparados entre todos os grupos analisados.

Contraria este achado menor número de préembriões "ótimos" em relação ao total de embriões mostrando fertilização normal (2PN) no grupo EDT. Talvez este fato possa ser conseqüência do critério falivel para classificação e seleção embrionária restritos somente ao dia de transferência $(D+3)$.

A seleção para transferência no dia $\mathrm{D}+3$ é praticada rotineiramente em muitos serviços de reprodução assistida ${ }^{20-22}$. Todavia, outros critérios importantes observados desde o momento da checagem da fertilização (cerca de 18 a 20 horas após a ICSI) até o momento da transferência têm sido demonstrados $23-25$.

Volpes et al..$^{22}$, tendo como opção a transferência máxima de três pré-embriões, e considerando "bons" aqueles com mais de oito blastômeros e menos de $20 \%$ de fragmentação, entre os três pré-embriões transferidos, obtiveram os seguintes resultados em relação à taxa de implantação e taxa de gravidez, respectivamente: transferência de três pré-embriões, sendo dois "bons", taxa de implantação $20,0 \%$ e taxa de gravidez $45,5 \%$; transferência de três pré-embriões, sendo três "bons", taxa de implantação 19,3\% e taxa de gravidez 39,5\%.

Comparando-se prognóstico e critérios para transferência embrionária, observa-se, por exemplo, que Zollner et al. ${ }^{26}$ emprega critérios de pronúcleos com uma estreita correlação para a formação de blastocistos viáveis. A morfologia alterada na fase de pronucleos parece também estar diretamente relacionada com maior indice de anormalidades genéticas embrionárias. Estu$\operatorname{dos}^{27,28}$ correlacionam as características do zigoto com os resultados de diagnóstico genético préimplantacional, demonstrando forte correlação entre os parâmetros analisados.

Donadio ${ }^{25}$ constatou uma correlação imperfeita, sugerindo perda da acuidade nos critérios pronucleares isolados. Neste estudo, taxa de implantação de $17 \%$ foi obtida após a transferência de três a quatro embriões selecionados exclusivamente pela classificação dos pronúcleos e esta porcentagem foi menor que a taxa de implantação obtida após a transferência de dois embriões selecionados por meio de um escore de PN associado à morfologia do dia +3 (27\%). Em consonância com outros estudos, reforça-se a necessidade da aplicação de escores acumulativos para a classificação e seleção embrionária após FIV.

Este estudo, corroborando outros autores ${ }^{8}$, sugere a indicação precoce das técnicas de reprodução assistida para todas as pacientes portadoras de EDT avançada que desejam a gravidez e não somente nos casos de pacientes com 38 anos ou mais, com mais de oito anos de infertilidade e na presença de fator masculino grave. A paciente portadora de EDT, apesar de ter o ovário menos responsivo e embriões de qualidade inferior, é capaz de conseguir a gestação por meio das técnicas de reprodução assistida, com boas chances de sucesso.

\section{Referências}

1. Sampson JA. Peritoneal endometriosis due to the menstrual dissemination of endometrial tissue into the peritoneal cavity. Am J Obstet Gynecol. 1927;14(2):422-69. 
2. Nisolle M, Donnez J. Peritoneal endometriosis, ovarian endometriosis, and adenomyotic nodules of the rectovaginal septum are three different entities. Fertil Steril. 1997;68(4):585-96.

3. Steptoe PC, Edwards RG. Birth after reimplantation of human embryo. Lancet. 1978;2(8085):366.

4. Palermo G, Joris H, Devroey P, Van Steirteghem AC. Pregnancies after intracytoplasmatic injection of single spermatozoon into an oocyte. Lancet. 1992;340(8810):17-8.

5. Nygren KG, Andersen AN. Assisted reproductive technology in Europe, 1999. Results generated from European registers by ESHRE. Hum Reprod. 2002;17(12):3260-74

6. Olivennes F, Feldberg D, Liu HC, Cohen J, Moy F, Rosenwaks Z. Endometriosis: a stage by stage analysis - the role of in vitro fertilization. Fertil Steril. 1995;64(2):392-8.

7. Azem F, Lessing JB, Geva E, Shahar A, Lerner-Geva L, Yovel I, et al. Patients with stages III and IV endometriosis have a poorer outcome of in vitro fertilization-embryo transfer than patients with tubal infertility. Fertil Steril. 1999;72(6):1107-9.

8. Barnhart K, Dunsmoor-Su R, Coutifaris C. Effect of endometriosis on in vitro fertilization. Fertil Steril. 2002;77(6):1148-55.

9. Tournaye H, Verheyen G, Albano C, Camus M, Van Landuyt L, Devroey P, et al. Intracytoplasmic sperm injection versus in vitro fertilization: a randomized controlled trial and a meta-analysis of the literature. Fertil Steril. 2002;78(5):1030-7.

10. Marconi G, Vilela M, Quintana R, Sueldo C. Laparoscopic ovarian cystectomy of endometriomas does not affect the ovarian response to gonadotropin stimulation. Fertil Steril. 2002;78(4):876-8.

11. Garcia-Velasco JA, Arici A. Surgery for the removal of endometriomas before in vitro fertilization does not increase implantation and pregnancy rates. Fertil Steril. 2004;81(5):1206.

12. Geber S, Prates LFVS, Ferreira DP, Sales L, Sampaio M. Resultados de técnicas de reprodução assistida em pacientes previamente submetidas a cirurgia ovariana para o tratamento da endometriose. Rev Bras Ginecol Obstet. 2002;24(6):371-6.

13.Zikopoulos K, Kolibianakis EM, Devroey P. Ovarian stimulation for in vitro fertilization in patients with endometriosis. Acta Obstet Gynecol Scand. 2004;83(7):651-5.

14. Revised American Society for Reproductive Medicine classification of endometriosis: 1996. Fertil Steril. 1997;67(5):817-21.

15. Veek L. Preembryo grading. In: Veek L, editor. Atlas of the human oocyte and early conceptus. Baltimore: Williams \& Wilkins; 1991. p. 121-49.

16. Svalander P, Forsberg AS, Jakobsson AH, Wikland M. Factors of importance for the establishment of a successful program of a intracytoplasmic sperm injection treatment for male infertility. Fertil Steril. 1995;63(4):828-37.

17.Borges Júnior E, Rossi-Ferragut LM. Cultura prolongada: quando indicar? In: Scheffer BB, Remohí J, Garcia-Velasco J, Pellicer A, Simón C, editores. Reprodução humana assistida. São Paulo: Atheneu; 2003. p. 177-88.

18. Adamson GD. Treatment of endometriosisassociated infertility. Semin Reprod Endocrinol. 1997;15(3):263-71.

19. Cunha-Filho JS, Gross JL, Lemos NA, Dias EC, Vettori D, Souza CA, et al. Prolactin and growth hormone secretion after thyrotrophin-releasing hormone infusion and dopaminergic (DA2) blockade in infertile patients with minimal/mild endometriosis. Hum Reprod. 2002;17(4):960-5.

20. Racowsky C, Jackson KV, Cekleniak NA, Fox JH, Hornstein MD, Ginsburg ES. The number of eightcell embryos is a key determinant for selecting day 3 or day 5 transfer. Fertil Steril. 2000;73(3):558-64.

21. Rienzi L, Ubaldi F, Iacobelli M, Ferrero S, Minasi MG, Martinez F, Tesarik J, Greco E. Day 3 embryo transfer with combined evaluation at the pronuclear and cleavage stages compares favourably with day 5 blastocyst transfer. Hum Reprod. 2002;17(7):1852-5.

22. Volpes A, Sammartano F, Coffaro F, Mistretta V, Scaglione P, Allegra A. Number of good quality embryos on day 3 is predictive for both pregnancy and implantation rates in in vitro fertilization/ intracytoplasmic sperm injection cycles. Fertil Steril. 2004;82(5):1330-6.

23. Jaroudi K, Al-Hassan S, Sieck U, Al-Sufyan H, AlKabra M, Coskun S. Zygote transfer on day 1 versus cleavage stage embryo transfer on day 3: a prospective randomized trial. Hum Reprod. 2004;19(3):645-8.

24. Desai NN, Goldstein J, Rowland DY, Goldfarb JM. Morphological evaluation of human embryos and derivation of an embryo quality scoring system specific for day 3 embryos: a preliminary study. Hum Reprod. 2000;15(10):2190-6.

25. Donadio NF. Influência nas taxas de implantação, da seleção de pré-embriões para transferência uterina a partir do escore dos pró-nucleados associado ao obtido no terceiro dia de cultivo [tese]. São Paulo: Santa Casa de São Paulo; 2004.

26.Zollner U, Zollner KP, Hartl G, Dietl J, Steck T. The use of a detailed zygote score after IVF/ICSI to obtain good quality blastocysts: the German experience. Hum Reprod. 2002;17(5):1327-33.

27. Gianarolli L, Magli MC, Ferraretti AP, Fortini D, Grieco N. Pronuclear morphology and chromosomal abnormalities as scoring criteria for embryo selection. Fertil Steril. 2003;80(2):341-9.

28. Borges EJ, Rossi LM, Farah L, Guilherme P, Rocha $\mathrm{CC}$, Ortiz V, et al. The impact of pronuclear orientation to select chromosomally normal embryos. J Assist Reprod Genet. 2005;22(3):107-14. 